\title{
ACTIVIDAD AGRARIA Y PAISAJE. LA GANADERIÁ, CLAVE EN LA CONSERVACIÓN DEL PAISAJE VASCO-ATLÁNTICO ${ }^{1}$
}

\author{
Eugenio Ruiz Urrestarazu \\ Rosario Galdos Urrutia \\ Departamento de Geografía, Prehistoria y Arqueología \\ Universidad del País Vasco/Euskal Herriko Unibertsitatea UPV/EHU \\ eugenio.ruiz@ehu.es, osario.galdos@ehu.es
}

\section{RESUMEN}

Se parte de la constatación de que el paisaje rural es en gran medida una manifestación territorial de la propia actividad agraria. Cualquier modificación en esta podría provocar cambios paisajísticos. El objetivo del artículo es doble: analizar y valorar la evolución reciente de la ganadería en el País Vasco y comprobar su impacto en el paisaje. Se deduce que, durante el último período intercensal 1999-2009, el declive pronunciado de la actividad ganadera no se ha traducido de forma generalizada en cambios paisajísticos profundos debido a causas achacables a la propia reconversión agraria y a otras causas socioeconómicas ajenas a la misma.

Palabras clave: Ganadería, Paisaje, País Vasco.

\section{ABSTRACT}

We start from the established fact that the countryside is largely a territorial manifestation of the agricultural activity. Any change to this could produce landscape alterations. The aim of this article is twofold: analyzing and assessing the recent evolution of the livestock in the Basque Country and seeing its impact on the landscape. It follows that during the censual period 1999-2009 the sharp decline of livestock farming has not rendered, generally speak-

Fecha de recepción: febrero 2012.

Fecha de aceptación: febrero 2013.

1 Este trabajo se inserta dentro del Proyecto de Investigación «Las unidades básicas de paisaje agrario en España: identificación, delimitación, caracterización y valoración. La España atlántica y Navarra» (Ministerio de Ciencia e Innovación. Referencia: CSO2009-12225-c05-04) 
ing, deep changes, owing to several causes depending on the farming reconversion itself and other socio-economical reasons far apart from the same.

Key words: Livestock, Landscape, Basque Country.

\section{INTRODUCCIÓN. EL PAISAJE VASCO-ATLÁNTICO}

El objeto de este artículo es doble. En primer lugar se pretende analizar y valorar la evolución reciente de la actividad ganadera en el País Vasco dentro de una situación generalizada de retracción y abandono del sector agroganadero. Como consecuencia del análisis anterior se quiere poner en relación los resultados constatados con la influencia que puedan ejercer sobre el mantenimiento o la transformación del paisaje del ámbito vasco-atlántico.

Para ello se parte de la constatación de que el paisaje rural es en gran medida una manifestación territorial de la propia actividad agraria. Tanto los elementos del paisaje como su organización y su propia evolución dependen de manera vinculante de las estructuras agrarias, los sistemas y tipos de cultivo y la orientación productiva de las explotaciones. Cualquier modificación en estos últimos factores se trasluce en cambios paisajísticos (Gascó et al., 2002). La relación entre paisaje y ganadería ha sido estudiada por numerosos autores desde diferentes perspectivas y ámbitos agroecológicos. A modo de ejemplo se pueden mencionar trabajos de carácter histórico y sobre ámbitos diversos como los de Corbera Millán (2006 y 2008), Cascos Maraña (2011), Elías Pastor y Fuentes Ochoa (2001), Llorente Pinto (2011), Silva Pérez (2010), Vila i Subirós et al. (2009) y Valle Buenestado (1995). También se ha investigado la influencia que sobre los paisajes ejerce la práctica política y en particular se ha demostrado en estudios de caso (Brady et el., 2012; Tzanopoulos et al., 2012) que el valor de los paisajes en mosaico es sensible a dichas prácticas.

El actual paisaje rural de los valles vasco-atlánticos, el que se podría denominar con toda propiedad como el paisaje del caserío vasco, mantiene una configuración sencilla, pero a la vez compleja, aunque esta contraposición pueda sonar paradójica. Sencilla porque son sólo tres los elementos esenciales de su paisaje: la dispersión del hábitat, los prados y las coníferas. Mientras que su complejidad le viene de la particular ordenación de los mismos, fruto de factores diversos entre los que se deben mencionar la intrincada topografía, la reducida base territorial del caserío y el predominio del trabajo a tiempo parcial. Todo ello da lugar a una estructura en mosaico de las laderas de los valles donde sus teselas principales son las parcelas de pinares, los prados, y en su caso, los rodales de bosque mixto atlántico. El conjunto resulta armonioso y atractivo a la vista, que disfruta de la combinación de formas y colores, presididos por las diferentes tonalidades del verde, desde el profundo casi negro de los pinares de repoblación hasta el verde claro y alegre de las praderías. No es necesario advertir que esta descripción es una simplificación que esconde variantes locales que se alejan de este cuadro general, variantes que a su vez se caracterizan por el grado de preeminencia en la combinación de los espacios cerrados y oscuros, los bosques de coníferas de rápido crecimiento, o de los prados de diente y siega, las áreas abiertas y claras. El predominio de los primeros está asociado a entornos rurales en que el abandono de las actividades agrarias ha desembocado en un aprovechamiento forestal del territorio, mientras que los segundos 
permanecen allí donde los caseríos siguen desempeñando una función ganadera significativa. Dicho en pocas palabras, la permanencia de este valioso paisaje en mosaico, de prados y manchas forestales, presidido siempre por las motas blancas dispersas de los caseríos, coronados por el rojo de sus techumbres, sólo es posible si estas explotaciones agrarias conservan su dedicación agraria y, más específicamente, ganadera. Porque el paisaje vasco-atlántico que hoy contemplamos es reflejo, en una parte sustancial aunque no única, de la actividad ganadera.

No hay que olvidar sin embargo que este paisaje actual del caserío es una construcción relativamente reciente, un estado evolutivo más dentro de una larga trayectoria histórica que ofrece fases diversas acordes con la estructura socioeconómica dominante en cada momento, tal como ha sido descrito y explicado por numerosos investigadores (entre otros, Caro Baroja, 1971; García Fernández, 1975; Etxezarreta, 1977; Ainz, 2001; Alberdi, 2001; Ruiz y Galdos, 2005). El caserío coetáneo, y aquí nos referimos a aquél que mantiene alguna actividad agraria ligada al mercado como fuente de financiación familiar, aunque sea parcial o secundaria, es heredero de las transformaciones provocadas por la reestructuración agraria que se inicia con la industrialización y se continúa en la fase postindustrial o posmoderna. Y su resultado, no exclusivo, es un caserío de orientación ganadera y forestal. La conjunción de ambas actividades da lugar al paisaje mixto de campiña atlántica. Pero esto no fue siempre así. En los albores de la primera industrialización y hasta bien avanzado el siglo XX el paisaje del caserío reflejaba una dedicación agrícola predominante, muy intensiva en el aprovechamiento del suelo y en trabajo familiar, donde el monte había perdido casi toda su cubierta arbórea en beneficio de landas y helechales como medio de mantener la fertilidad de los predios al ser transformados en fiemo junto con las deyecciones de un ganado vacuno lo suficientemente rústico y versátil que proporcionase leche, carne y, muy importante, fuerza animal para las labores propias de una explotación que giraba en torno a la producción agrícola. Pero en otra fase precedente, que se puede remontar a la Edad Media y que perdura hasta el siglo XVIII, la importancia y los cuidados del monte arbolado eran estratégicos en una sociedad que basaba una parte sustancial de su riqueza en la producción siderúrgica centrada en las ferrerías, establecimientos industriales esparcidos por el territorio y muy enraizados en el medio rural del que extraían la energía hidráulica de los ríos y el combustible de los hornos en forma de carbón vegetal. En esta economía el bosque y el trabajo asociado de los labradores, que además eran arrieros, resultaba fundamental. A estas dos fases históricas sucintamente evocadas, una más antigua agrícola, forestal y ferrona, y otra posterior de carácter agrícola predominante, le ha sucedido la actual, ganadera y maderera (Michel, 2004). Cualquier cambio en la orientación técnico económica de las explotaciones contemporáneas, o en el caso más extremo su propia supervivencia, modificará la organización territorial del espacio rural y su paisaje. Aunque para el estudio concreto del abandono, que no es objeto central de este artículo, existen otras variables que deben tenerse en cuenta, tal como ponen de manifiesto Hatna y Bakker (2011)

Al ser la ganadería la actividad garante del mantenimiento del paisaje de la campiña atlántica vasca, la evolución de la misma resulta crucial para el devenir de la configuración del territorio. Para su análisis se han utilizado como fuentes los resultados de los dos últimos censos agrarios, 1999 y 2009. Dado que los criterios empleados en uno y otro censo difieren, debido a que el más reciente utiliza un universo de explotaciones más restringido al referirse 
sólo a las propiamente agrícolas y quedando excluidas las netamente forestales, se ha intentado equiparar sus datos para que sean comparables. Para ello se ha contado con los datos que del País Vasco y de sus tres provincias ofrece el EUSTAT (Instituto Vasco de Estadística) en unas tablas comparativas de los tres últimos censos agrarios. En la elaboración de los mapas municipales se han utilizado los datos censales ofrecidos por el INE, optando por analizar únicamente las explotaciones con SAU (Superficie Agrícola Utilizada). Se advierte que los resultados censales que proporcionan ambos organismos estadísticos, INE y EUSTAT, no siempre coinciden.

\section{UNA ACTIVIDAD GANADERA EN CLARA DECADENCIA}

Desde los inicios de la Política Agraria Común (P.A.C.) se apostó en Europa por un redimensionamiento de las explotaciones de manera que al aumentar estas su capacidad productiva gracias a la intensificación o a la expansión de su base territorial garantizase su supervivencia al hacerse más competitivas en un mercado abierto y crecientemente global (Piorr et al., 2009; Naredo, 1996). Sólo aquellas explotaciones que fuesen capaces de encarar esta reestructuración serían capaces de mantenerse y de hecho muchas explotaciones fueron abandonadas. Diversos autores pensaban que este proceso de abandono y reestructuración sería temporal hasta que se estableciese un nuevo equilibrio basado en un menor número de explotaciones pero más productivas y competitivas (Domínguez, 1996, Sineiro et al., 2006, Camós, 2006). Cuando se comprobó que dicho proceso de abandono no se mostraba coyuntural sino que se mantenía en el tiempo, se fomentaron otras políticas agrarias más dependientes de factores medioambientales ligados a la extensificación y a los productos de calidad. Autores como Arnalte et al. (2012) han puesto de manifiesto el influjo que han ejercido las políticas aplicadas, en particular la PAC, sobre espacios rurales, tanto desde perspectivas globales (Cejudo y Maroto, 2010; Bournaris y Manos, 2012) como desde ámbitos más concretos como las zonas de montaña (Malagón, 2012) o las producciones ganaderas (Berga et al., 2008).

Sin embargo, y a pesar de que estas últimas políticas han dado resultados positivos en un cierto número de explotaciones, el abandono sigue vigente. En otros casos no se produce un abandono propiamente dicho sino una disminución de la actividad a través de la agricultura a tiempo parcial que en mucho casos desemboca en un agricultura que cabe denominar de ocio (Ainz, 1999). También han detectado otros autores que «el abandono del suelo agrario se presenta, al menos en sus estadio iniciales, oculto bajo formas de infrautilización del mismo, entendiendo por tal, la de tierras dedicadas a praderas y pastizales con bajo nivel de aprovechamiento, alto riesgo de degradación y de pérdida de valor» (Murua et al., 2012). Correspondería a la fase que Keenleyside y Tucker (2010, p. 10) denominan semi-abandonment o hidden abandonment (semiabandono o abandono oculto).

En el caso concreto del País Vasco desde 1989 a 2009 desaparecen 10.735 explotaciones. En cifras relativas esta disminución supone una pérdida del $40 \%$ de las explotaciones en veinte años. Estas cifras son elocuentes de que el proceso de abandono dista mucho de ir cesando (Ruiz Urrestarazu y Galdos Urrutia, 2011). Y lo que es aún más significativo, las dos terceras partes de los abandonos se producen en el decenio más reciente. Por tanto el proceso se intensifica hasta el punto que algunos autores han llegado a especular desde una 
perspectiva económica sobre el coste de un cese total de la agricultura en el país (Murua, et al., 2006).

Si nos ceñimos a las explotaciones ganaderas, que son el objetivo de este trabajo, se observa la misma tendencia. Y el abandono de estas explotaciones se acompaña de un descenso en la cabaña. Es decir que el cese de unas no se compensa con la intensificación de otras.

Tabla 1

EVOLUCIÓN DEL NÚMERO DE EXPLOTACIONES Y DE CABEZAS DE GANADO MAYOR EN EL PAÍS VASCO

\begin{tabular}{|l|c|c|c|c|}
\hline & \multicolumn{2}{|c|}{1999} & \multicolumn{2}{c|}{2009} \\
\hline & $\mathrm{N}^{\mathrm{o}}$ explotaciones & $\mathrm{N}^{\mathrm{o}}$ cabezas & $\mathrm{N}^{\mathrm{o}}$ explotaciones & $\mathrm{N}^{\mathrm{o}}$ cabezas \\
\hline Bovinos & 10.352 & 179.328 & 5.946 & 135.448 \\
\hline Ovinos & 4.790 & 313.896 & 4.546 & 271.433 \\
\hline Caprinos & 1.778 & 19.402 & 1.616 & 21.549 \\
\hline Porcinos & 2.891 & 38.067 & 902 & 16.102 \\
\hline Equinos & 4.298 & 15.366 & 3.504 & 19.249 \\
\hline
\end{tabular}

Fuente: EUSTAT. Censos Agrarios

En diez años se han suprimido más de 100.000 cabezas de ganado mayor. Este descenso no ha afectado a todas las cabañas ya que se ha centrado en aquellas que suponen un mayor peso numérico y económico, la porcina, la bovina y la ovina. En este análisis nos vamos a centrar en los ganados bovino y ovino ya que además de tener una mayor repercusión económica y social son las que mayor influencia han tenido en la conformación del paisaje rural, sobre todo en los valles atlánticos.

Si durante el período analizado un tercio del total de las explotaciones agrarias $(33,1 \%)$ ha desaparecido, el porcentaje de explotaciones de bovino que cesan su actividad supera a las anteriores en más de 10 puntos (42,6\%). La cifra es bien expresiva de la crisis de supervivencia en que están abocadas buena parte de estas empresas ganaderas. Sin embargo el número de cabezas de bovino no ha menguado en un porcentaje equiparable, sino que su caída ha sido menor. De cada cuatro cabezas que se registraban en 1999, sólo se han mantenido tres en 2009. No obstante, el número de bovinos por explotación crece de 16,8 en el año 1999 al 22,8 en 2009. Pero lo importante cara a su impacto sobre el territorio es reseñar esta notable mengua ganadera.

A diferencia del bovino el descenso de explotaciones y cabezas de ganado ovino ha sido considerablemente menor, aunque también se observa en esta década una clara tendencia al abandono. Las explotaciones se han reducido un 5\% y la cabaña un 13\%. El mayor decrecimiento de esta última, de la cabaña ovina, ocasiona un descenso del número de cabezas por explotación que pasa de 65,5 en 1999 a 59,5 en 2009. En el caso del sector porcino han desaparecido algo más de los dos tercios de las explotaciones y algo más de la mitad de su cabaña, aunque ha aumentado el tamaño medio de las explotaciones que quedan al pasar de 13,2 cabezas en 1999 a casi 18 en el año 2009. También han desaparecido explotaciones 
dedicadas al sector caprino y equino si bien en estos dos sectores ello no ha venido acompañado de un descenso de sus cabañas, con lo que se ha mejorado el tamaño medio de las explotaciones que pasan de 10,9 a 13,3 en el caso del caprino y de 3,6 a 5,5 en el del equino.

Aunque la consideración de estos datos cuantitativos es necesaria para analizar su impacto sobre el territorio, se debe tener en cuenta también otros factores de carácter más cualitativo como el sistema de explotación del ganado que presenta diferentes grados de aprovechamiento del territorio en función de las características propias de cada especie y su orientación productiva. Aunque más adelante se hablará de ello, cabe por ejemplo señalar a modo introductorio que el bovino de orientación láctea, o mejor dicho, el sistema de producción utilizado es el más independiente de los recursos propios de la explotación; por el contrario el bovino de aptitud cárnica, explotado bajos sistemas más extensivos, aparece más ligado al aprovechamiento directo de la producción herbácea de la propia explotación. Estas diferencias, con todas las consecuencias ecológicas, laborales y sociales que conllevan, son así percibidas por los propios ganaderos y sus sindicatos representativos (Baserri Bizia, 2011). La diferencia es aún más clara en el caso del ganado ovino cuyo manejo se basa en el pastoreo.

\section{EL IMPACTO TERRITORIAL: LA DENSIDAD GANADERA}

La dependencia del paisaje respecto de la ganadería será tanto mayor cuanto mayor sea la intensidad ganadera sobre el territorio. Aunque los insumos alimenticios de una explotación, o una parte de los mismos, pueden provenir de ámbitos ajenos a su propio terreno e incluso de áreas más lejanas, en el caso del País Vasco la ligazón entre actividad pecuaria y utilización de la SAU para alimento del ganado es patente. Por esta razón se sostiene que el mantenimiento de la campiña atlántica actual se basa en la continuidad de la orientación ganadera. El declive ya comprobado del número de explotaciones y de cabezas de ganado debería tener impactos diferenciados según la intensidad del proceso. Por este motivo interesa conocer cuál es la distribución territorial de la ganadería y su evolución temporal en el último período intercensal. Para su muestra cartográfica se ha utilizado las UGM totales como unidad de cuenta ganadera, la escala municipal como ámbito espacial y la SAU como territorio de referencia. La utilización de esta última como referencia territorial tiene la ventaja respecto de otras alternativas, como las áreas de pasto permanente, de que permite discriminar mejor la situación de ambas vertientes del País Vasco, la atlántica y la mediterránea. En la vertiente costera la superficie de pastos permanentes casi coincide con la SAU ya que en esta última su dedicación se encuentra en su mayor parte al servicio de la ganadería. Los resultados apenas diferirían si se utilizasen cualquiera de los dos espacios de referencia, SAU o pastos permanentes. Sin embargo la situación es muy diferente en la vertiente interior que se caracteriza por una orientación más agrícola, sobre todo en las comarcas centrales y meridionales. Aquí la SAU es mucho más extensa y la superficie dedicada a alimento del ganado sólo ocupa una reducida porción de la misma. Por consiguiente la densidad ganadera se reduce considerablemente al emplear este índice.

En la Figura 1 correspondiente al año 1999 se diseñan con claridad las pautas de distribución territorial del conjunto del ganado mayor. La principal es la dicotomía entre ambas vertientes. La vertiente atlántica detenta una intensidad ganadera considerable en comparación 


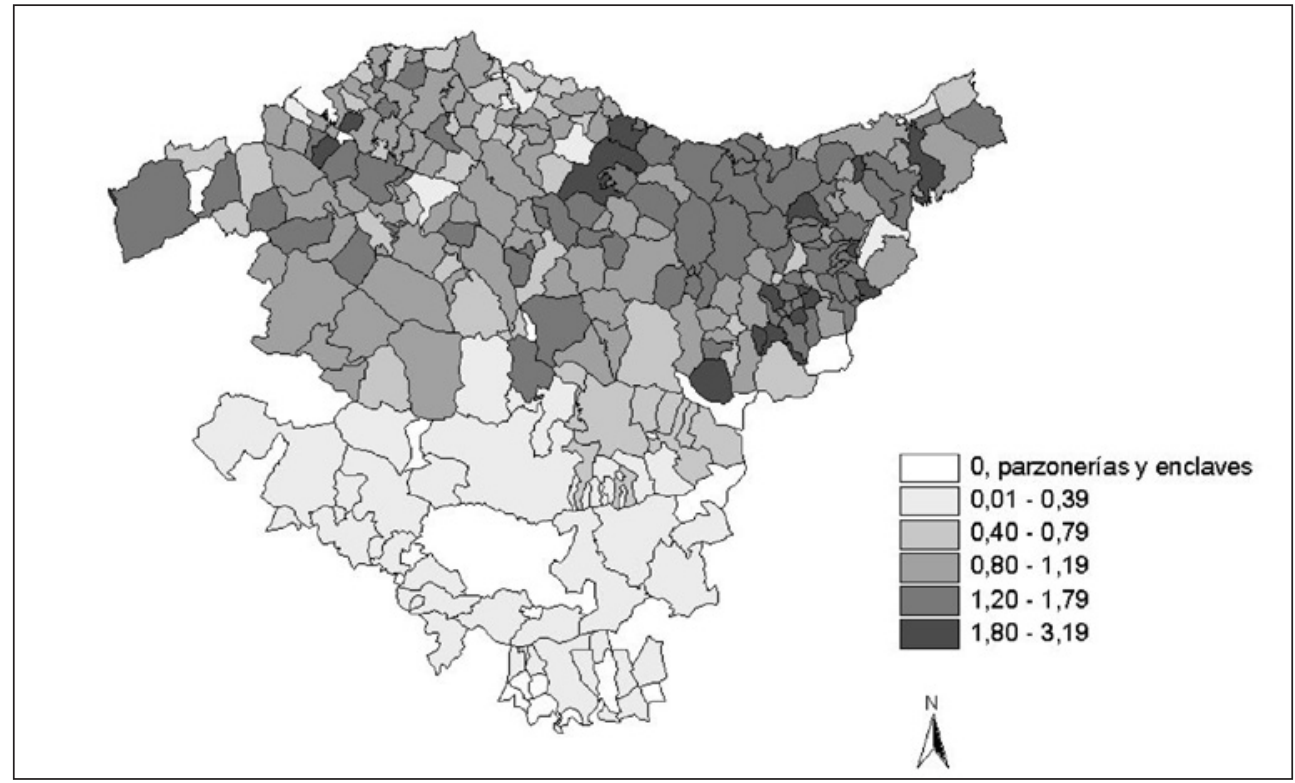

Fuente: INE, Censo Agrario 1999.

con la mediterránea. Es decir que se produce una gradación de densidad decreciente hacia el mediodía, hasta el punto de que en algunos municipios riojanos-alaveses ni siquiera hay presencia alguna de UGM. Aunque lo dicho es cierto en términos generales conviene hacer un par de puntualizaciones. La primera consiste en que el área de fuerte densidad, que abarca las provincias de Bizkaia y Gipuzkoa y las comarcas cantábricas de Álava, desborda la línea divisoria atlántico-mediterránea para adentrarse hacia el sur por las comarcas alavesas de Estribaciones del Gorbea y el norte de la Llanada. En efecto estas zonas citadas evolucionaron a partir del inicio de la profunda reestructuración agraria de los años 1960 desde una dedicación más agrícola hacia otra en que la ganadería pasaba a ser la orientación dominante. A resultas de este cambio productivo su paisaje sufrió una transformación que se podría definir como un proceso de «atlantización», en el cual las parcelas de cultivos herbáceos anuales pasaron a ser ocupadas por praderas permanentes. La segunda aclaración se refiere a que dentro del área atlántica la intensidad es superior en la provincia oriental de Gipuzkoa.

Diez años más tarde la Figura 2 sigue conservando los rasgos generales descritos, pero al mismo tiempo se detectan algunas modificaciones que merece reseñar. Se detecta una ausencia de pautas territoriales claras que puedan ser observadas a esta escala. De hecho en el conjunto del País Vasco existe casi un equilibrio entre el número de municipios que pierden intensidad ganadera (129) y el número de los que la incrementan (116). Y este equilibrio se repite en ambas vertientes y en las tres provincias. Como media regional el número de UGM por hectárea de SAU ha registrado un aumento de 0,73 en 1999 a 0,79 en 2009. Pero si se compara la superficie total regional que gana o pierde intensidad los resultados son más 


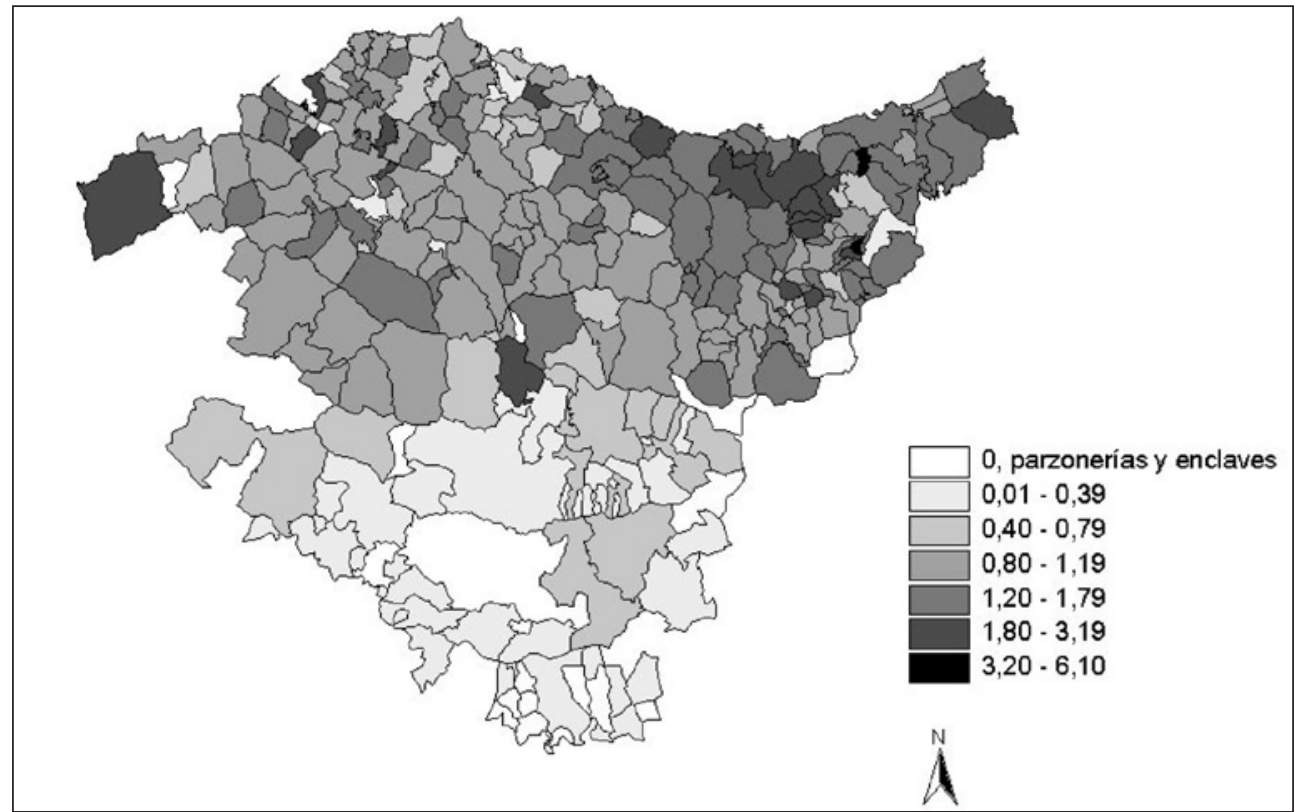

Fuente: INE, Censo Agrario 2009.

Tabla 2

EVOLUCIÓN DE LA SUPERFICIE AGRÍCOLA UTILIZADA ENTRE 1999 Y 2009

\begin{tabular}{|l|c|c|c|}
\hline & 1999 & 2009 & $1999=100$ \\
\hline Álava & 131.963 & 99.761 & 75,6 \\
\hline Bizkaia & 64.468 & 46.459 & 72,1 \\
\hline Gipuzkoa & 58.859 & 55.237 & 93.8 \\
\hline País Vasco & 255.290 & 201.457 & 78,9 \\
\hline
\end{tabular}

Fuente: EUSTAT, Censos Agrarios.

contrastados. Los 120 municipios que pierden intensidad ganadera representan el 36,6\% de la superficie regional, mientras que los 116 que ganan intensidad suponen el 54,6\%. Esto significa que, sin tener en cuenta los municipios que no sufren modificación a lo largo del período intercensal, más de la mitad del territorio experimenta una mayor presión ganadera.

Para valorar estos resultados estadísticos en relación con su influencia en los cambios de uso del suelo y en el paisaje es necesario efectuar algunas precisiones. En la mayoría de los casos ese aumento de la intensidad ganadera no se debe al incremento del número de efectivos de la cabaña, sino a la merma de la SAU. La figura 3 señala con clara nitidez el descenso 


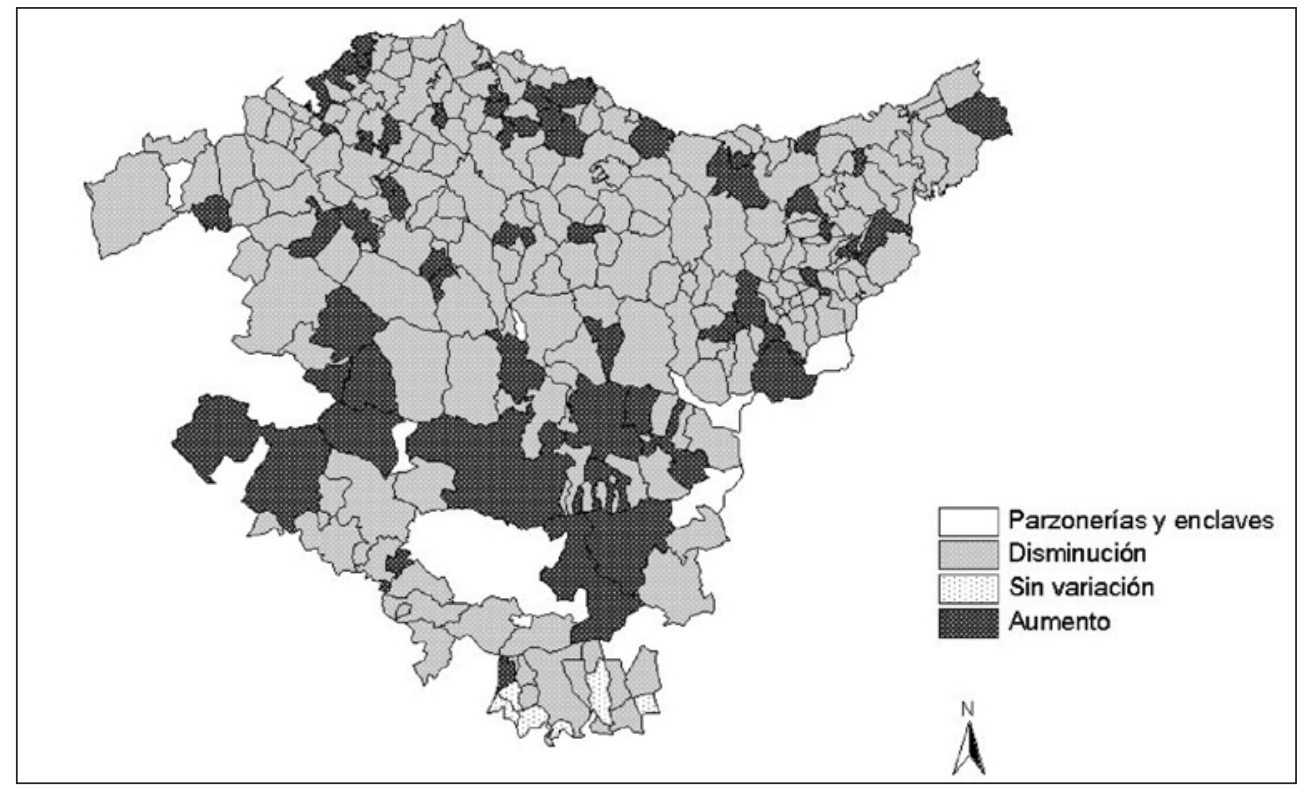

Fuente: INE, Censos Agrarios.

generalizado de UGM en el conjunto del País Vasco, más palpable en la vertiente atlántica. Únicamente las comarcas centrales de Álava manifiestan un crecimiento que también se observaba en el anterior mapa de intensidad ganadera. Cabe interpretar este hecho como un cierto desplazamiento hacia el sur del interés por la ganadería. Por tanto, si desciende en el País Vasco el número de UGM y a su vez aumenta la densidad ganadera la clave está en la retracción de la SAU. En efecto, la disminución de esta superficie ha sido en el País Vasco del $21 \%$ entre los años de referencia según datos del EUSTAT, siendo mayor en Bizkaia y más moderada en la provincia guipuzcoana.

Estos resultados conducen a tres conclusiones, las dos primeras de carácter metodológico:

a) Debido a los problemas de comparación estadística que genera la diferente metodología utilizada en cada censo, los resultados numéricos absolutos deben ser tratados con cautela; de hecho, la pérdida de SAU en el País Vasco llega al 26,6\% si se manejan los datos censales aportados por el INE. Aún así, ambas fuentes manifiestan una clara tendencia regresiva de la SAU.

b) Para la correcta interpretación del cálculo de la intensidad ganadera es fundamental el indicador que se utilice, que podrá variar en cada caso de estudio en función de las características propias del territorio. En unos casos el espacio de referencia más adecuado será la superficie de dedicación ganadera, en otros la SAU o en otros la superficie total.

c) El descenso constatado en el número de UGM en el País Vasco parece estar ya manifestándose en la organización territorial, sobre todo en la vertiente atlántica, a través de una retracción de la SAU y por tanto en la configuración paisajística. 


\section{LA ORIENTACIÓN PRODUCTIVA DEL GANADO BOVINO}

La orientación productiva, lechera o cárnica, y su evolución tienen una influencia considerable en el aprovechamiento y manejo de las tierras y por tanto en el paisaje. Las vacas de aptitud lechera tienden a ser criadas estabuladas o semiestabuladas y una parte de su alimentación, en ocasiones la principal, proviene de piensos adquiridos fuera de la explotación. En consecuencia su arraigo en el territorio y sus recursos es mucho menor. Por el contrario el ganado de carne se maneja de forma más extensiva y aprovecha de forma directa, a diente, los herbazales de la explotación. En el País Vasco su dependencia del territorio es clara.

Tabla 3

EVOLUCIÓN DEL GANADO BOVINO DE APTITUD LÁCTEA EN EL PAÍS VASCO, 1999-2009

\begin{tabular}{|l|c|c|c|c|}
\hline \multirow{2}{*}{} & \multicolumn{2}{|c|}{1999} & \multicolumn{2}{c|}{2009} \\
\cline { 2 - 5 } & $\mathrm{N}^{\circ}$ explotaciones & $\mathrm{N}^{\mathrm{o}}$ cabezas & $\mathrm{N}^{\circ}$ explotaciones & $\mathrm{N}^{\circ}$ cabezas \\
\hline Vacas lecheras & 2.896 & 38.909 & 932 & 25.219 \\
\hline
\end{tabular}

Fuente: INE, Censos Agrarios.

La tendencia que ya se había detectado y estudiado en décadas precedentes sobre el cambio de muchas explotaciones de una dedicación láctea a otra cárnica se ha incrementado de manera espectacular en el último periodo intercensal. El precio percibido por los ganaderos vascos por cada litro de leche ha sido y sigue siendo la principal causa de la falta de viabilidad de las explotaciones junto con «la falta de autonomía del sector lácteo vasco (el que más gasto tiene en compras de alimento de toda Europa) en el que entre un $45 \%$ y un $60 \%$ de los gastos totales de la explotación, son de alimentación comprada» (Baserri Bizia, 2012, p. 12-13). La creciente subida del precio de las materias primas utilizadas para los piensos, sobre todo desde el año 2008, agrava la situación de las explotaciones dependientes de las compras de alimentación exterior. La baja rentabilidad de muchas explotaciones empuja a sus propietarios a conceder cada vez más protagonismo en la renta familiar a los ingresos provenientes de otras actividades no agrarias. Por tanto el tiempo activo que se dedica a la ganadería se resiente y al ser la ganadería láctea mucho más exigente en trabajo, una alternativa ampliamente difundida ha sido la transformación productiva hacia la cría de ganado de carne. Esta decisión acarrea además menores inversiones corrientes ya que el manejo del ganado se extensifica, basándose en el máximo aprovechamiento de los recursos propios de la explotación. La disminución de los gastos y el ahorro de trabajo compensan, al menos en parte, la menor rentabilidad de una explotación cárnica. Esta evolución que parece positiva desde una perspectiva medioambiental y paisajística no lo es tanto si se considera, como han advertido algunos autores, que en no pocos casos esta reconversión hacia la producción cárnica supone un paso intermedio para el abandono definitivo de la actividad ganadera, al menos como actividad generadora de renta (Alberdi, 2002).

Tal como ya se ha adelantado, la propensión al cambio productivo ha sido extraordinaria en el decenio 1999-2009. Si el decremento del número de explotaciones de bovino en general ha sido, como ya se ha señalado, del $42,6 \%$, el que se ha producido en las explotaciones 
con vacas lecheras se eleva a casi el 68\%, 26 puntos más. Esta enorme pérdida en el número explotaciones de leche no se corresponde de igual medida en la desaparición de cabezas de aptitud láctea. La mengua en el número de vacas lecheras ha sido del 35,2\%, cifra que no deja de ser asimismo apreciable. Por otro lado podría pensarse que el descenso de la cabaña láctea se compensaría con un aumento del ganado de otras aptitudes, pero tal cosa no ha sucedido. El número de cabezas que no son vacas lecheras también ha disminuido. En definitiva, en 2009 había menos cabezas de ganado bovino que en 1999 en cualquiera de las orientaciones productivas.

Si un número considerable de explotaciones lácteas han desaparecido pero el descenso en el número de cabezas no ha sido tan grande, la conclusión es obvia: las explotaciones que permanecen han adquirido una mayor intensidad ganadera.

Tabla 4

EVOLUCIÓN DEL NÚMERO DE EXPLOTACIONES Y DE VACAS LECHERAS SEGÚN TAMAÑO DE SAU EN EL PAÍS VASCO

\begin{tabular}{|l|c|c|c|c|c|c|}
\hline & \multicolumn{3}{|c|}{ Explotaciones lecheras } & \multicolumn{3}{c|}{ Vacas lecheras } \\
\hline Ha & 1999 & 2009 & $1999=100$ & 1999 & 2009 & $1999=100$ \\
\hline $1-5$ & 1.134 & 308 & 27,2 & 4.492 & 1.550 & 34,5 \\
\hline $5-10$ & 727 & 178 & 24,5 & 6.592 & 2.004 & 30,4 \\
\hline $10-20$ & 600 & 186 & 31,0 & 10.522 & 4.758 & 45,2 \\
\hline$>20$ & 435 & 260 & 59,8 & 17.303 & 16.906 & 97,7 \\
\hline Total & 2.896 & 932 & 32,2 & 38.909 & 25.218 & 64,8 \\
\hline
\end{tabular}

Fuente: INE, Censos Agrarios.

Ya se había visto que la disminución del número total de explotaciones con vacas lecheras ha disminuido de forma drástica. Pero su distribución por tamaño de la explotación no ha variado de modo sustancial. En ambos años censales el mayor número de explotaciones se concentra en las de menor tamaño de SAU, entre 1 y 5 Ha. Esto indica que todavía persiste un número comparativamente elevado de pequeñas explotaciones que mantienen su dedicación láctea. También conviene recordar que el caserío medio de la vertiente atlántica del País Vasco viene a contar con una SAU de unas 4-5 Ha por lo que buena parte de los mismos se encuentra dentro de esta categoría y, como ya es sabido, la dedicación prioritaria del mismo es la ganadera. Asimismo, no es aventurado suponer que en muchos caso se trata de explotaciones marginales, más ligadas a la agricultura de ocio que a la de mercado y más preocupadas por mantener limpios los prados de la invasión de maleza y matorral que por su rentabilidad inmediata, cuestión sobre la que más adelante se volverá. En cualquier caso la proporción de explotaciones en los intervalos menores de $20 \mathrm{Ha}$ ha disminuido en todos los casos, mientras que el único tramo que incrementa su representación relativa corresponde a las de mayores dimensiones. Donde se observa un cambio estructural es en el reparto de las cabezas por tamaño de explotación. Las explotaciones con más de 20 Ha acogían en 1999 al 44,5\% 
de la cabaña lechera. Diez años después esa proporción se eleva al 67\%. El fenómeno de la concentración productiva en explotaciones mejor dimensionadas es nítido. En contrapartida las explotaciones pequeñas y aún medias cada vez representan menor capacidad productiva, siempre en términos comparativos, porque el número medio de vacas por explotación ha aumentado en todos los intervalos de extensión. Es decir que independientemente de su tamaño, todas las explotaciones que se mantienen han aumentado su número de cabezas. Bien es cierto que en una medida muy liviana las menores de $10 \mathrm{Ha}$ y de una forma ya más manifiesta las de 10-20 Ha (de 17,5 vacas de media a 25,6). Y el salto cualitativo afecta a las más grandes: las explotaciones mayores de $20 \mathrm{Ha}$ pasan de 39,8 vacas de media por explotación a 65 . Se comprueba, por tanto, el proceso de abandono/intensificación. El cuadro siguiente es muy expresivo de dicho proceso, además de mostrar un reparto territorial muy diferenciado.

Tabla 5

EVOLUCIÓN DEL NÚMERO MEDIO DE VACAS LECHERAS ENTRE LAS EXPLOTACIONES CON SAU, 1999-2009

\begin{tabular}{|c|c|c|c|}
\hline & Álava & Bizkaia & Gipuzkoa \\
\hline 1999 & 25,3 & 11,2 & 13.4 \\
\hline 2009 & 60.9 & 22,5 & 23,7 \\
\hline
\end{tabular}

Fuente: INE, Censos Agrarios.

En las provincias litorales de Bizkaia y Gipuzkoa el número medio de vacas lecheras por explotación se ha duplicado en el decenio 1999-2009, mientras que en Álava el multiplicador ha sido del 2,4. Está claro que en esta provincia la evolución hacia explotaciones mejor dimensionadas desde el punto de vista productivista ha sido más avanzada, debido en gran medida a que su punto de partida estructural resultaba más propicio gracias a unas explotaciones originales con mayor base territorial. Ello ha dado lugar a unas empresas agrarias menos numerosas pero más dinámicas y productivas.

Por último el declive en el País Vasco del ganado bovino en su conjunto se puede contemplar en la Figura 4. La sencillez de su confección, que sólo representa el aumento o disminución de UG de bovino en cada municipio, facilita una visión rápida pero eficaz del conjunto del país. La escala municipal adoptada permite calibrar su repercusión territorial y por tanto detectar las áreas potencialmente susceptibles de sufrir modificaciones en los usos del suelo, la organización del espacio y en el paisaje. El decremento de la cabaña bovina es un hecho generalizado: 188 términos municipales manifiestan pérdidas, frente a sólo 47 que tienen ganancias. Otro aspecto interesante a considerar es que la mengua afecta de un modo más frecuente a las provincias costeras, que son a su vez las más ganaderas, pero que al mismo tiempo presentan mayores dificultades para la ampliación de sus estructuras agrarias y donde la separación entre lo urbano y lo rural aparece más difusa y las oportunidades de otros empleos no agrarios mejor repartidas espacialmente. En la provincia alavesa por el contrario se concentran buena parte de los municipios que ven incrementar su cabaña bovina fomentada por ganaderos más profesionales con mejores dotaciones estructurales tal como refleja la tabla 5 . 
Figura 4

VARIACIÓN DEL NÚMERO DE UG DE BOVINO 1999-2009

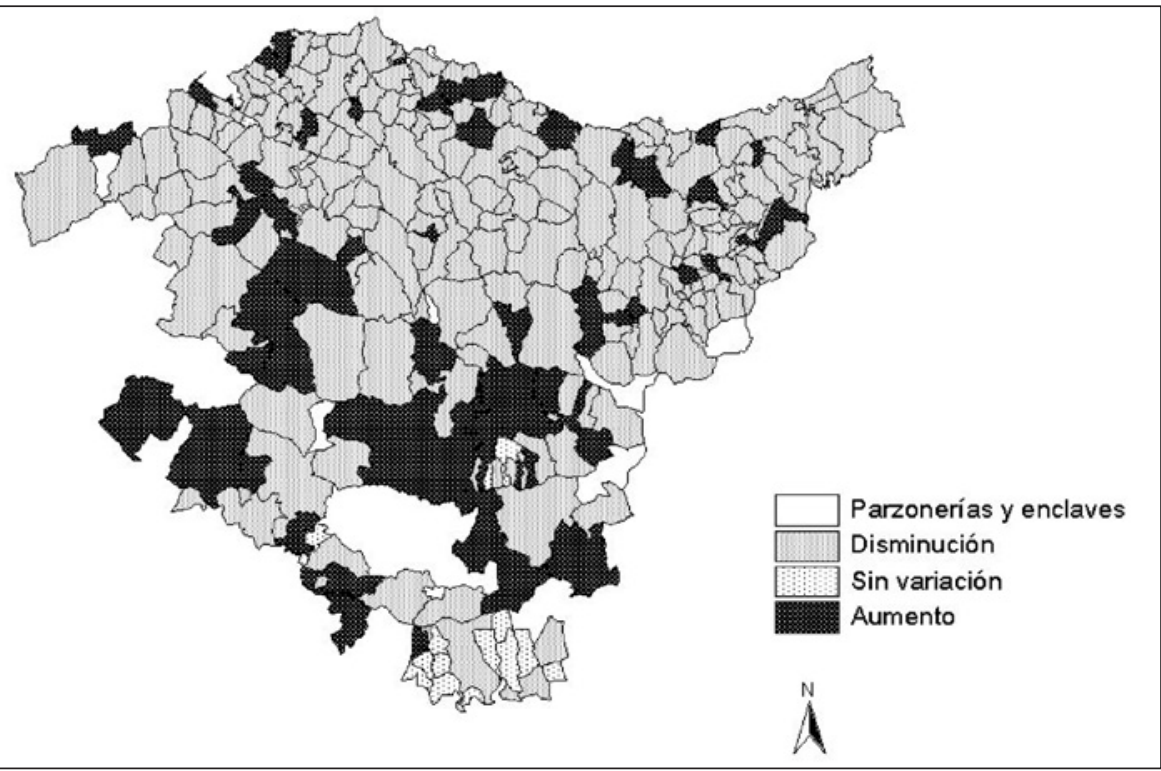

Fuente: INE, Censos Agrarios.

\section{UNA CABAÑA OVINA MÁS RESISTENTE AL ABANDONO}

Las explotaciones y la cabaña ovina también han disminuido pero con una intensidad mucho menos acusada que las bovinas. En la Tabla 3 se reflejaba que el número de explotaciones sólo había descendido 5,1 puntos porcentuales, mientras las cabezas lanares habían descendido 13,5 puntos. En principio, el mantenimiento de los pastos para este ganado, esencialmente montaraz en su variedad latxa, de aptitud lechera para la fabricación de quesos, ampliamente mayoritaria en el país, quedaría garantizado mientras el declive no se muestre más acusado.

Tabla 6

EVOLUCIÓN DEL NÚMERO DE EXPLOTACIONES Y DE CABEZAS DE GANADO OVINO SEGÚN TAMAÑO DE LA SAU EN EL PAÍS VASCO.

\begin{tabular}{|l|c|c|c|c|c|c|}
\hline & \multicolumn{3}{|c|}{ Explotaciones de ovino } & \multicolumn{3}{c|}{ Cabezas de ovino } \\
\hline $\mathrm{Ha}$ & 1999 & 2009 & $1999=100$ & 1999 & 2009 & $1999=100$ \\
\hline $1-5$ & 2.187 & 2.224 & 101,7 & 67.607 & 51.703 & 76,5 \\
\hline $5-10$ & 973 & 839 & 86,2 & 56.982 & 38.916 & 68,3 \\
\hline $10-20$ & 679 & 537 & 79,1 & 60.468 & 42.451 & 70,2 \\
\hline$>20$ & 467 & 640 & 137,0 & 106.014 & 131.222 & 123,8 \\
\hline Total & 4.306 & 4.240 & 98,5 & 291.071 & 264.292 & 90,8 \\
\hline
\end{tabular}

Fuente: INE, Censos Agrarios. 
El diferente comportamiento de los ganaderos de ovino respecto de los de bovino, especialmente del bovino lácteo, no sólo se refiere a una mayor resistencia al abandono a causa en gran medida a los menores costes de explotación sino también a la evolución reciente en sus explotaciones. Así, las explotaciones ovinas con menor superficie de SAU (1-5 Ha) son más numerosas en 2009 que diez años antes. Es decir que se han creado nuevas explotaciones ovinas, cerca de un $2 \%$ respecto al primer año censal, sobre bases territoriales reducidas. También a diferencia del vacuno, las explotaciones mejor dimensionadas, las que cuentan con más de $20 \mathrm{Ha}$ de SAU, son las que más han aumentado. En concreto durante los diez años contemplados se han creado 173 nuevas explotaciones ovinas, un $37 \%$ más. Por el contrario son las explotaciones medias, entre 5 y $20 \mathrm{Ha}$, las que han registrado una disminución de sus efectivos. A pesar de estos incrementos en los intervalos extremos, las únicas explotaciones que han aumentado su número de cabezas son las más grandes. De nuevo se constata el efecto de concentración de los efectivos ganaderos. Pero como contraste a este hecho también se constata que los rebaños son de media más pequeños, sea cual sea la superficie de sus explotaciones. De nuevo se manifiesta una dicotomía territorial entre las provincias costeras, con hatos de tamaño pequeño, entre las 36 unidades de Bizkaia y las casi 70 de Gipuzkoa, y la provincia alavesa con 125 cabezas de media por explotación. Las mayores dimensiones de las explotaciones de este territorio y la abundancia de pastos comunales que complementan las tierras particulares de las explotaciones ayudan a explicar las diferencias de magnitud.

La variación intercensal del número de UG de ovino está representada en la Figura 5. Son 142 los municipios que han perdido unidades ganaderas y 98 los que las han incrementado.

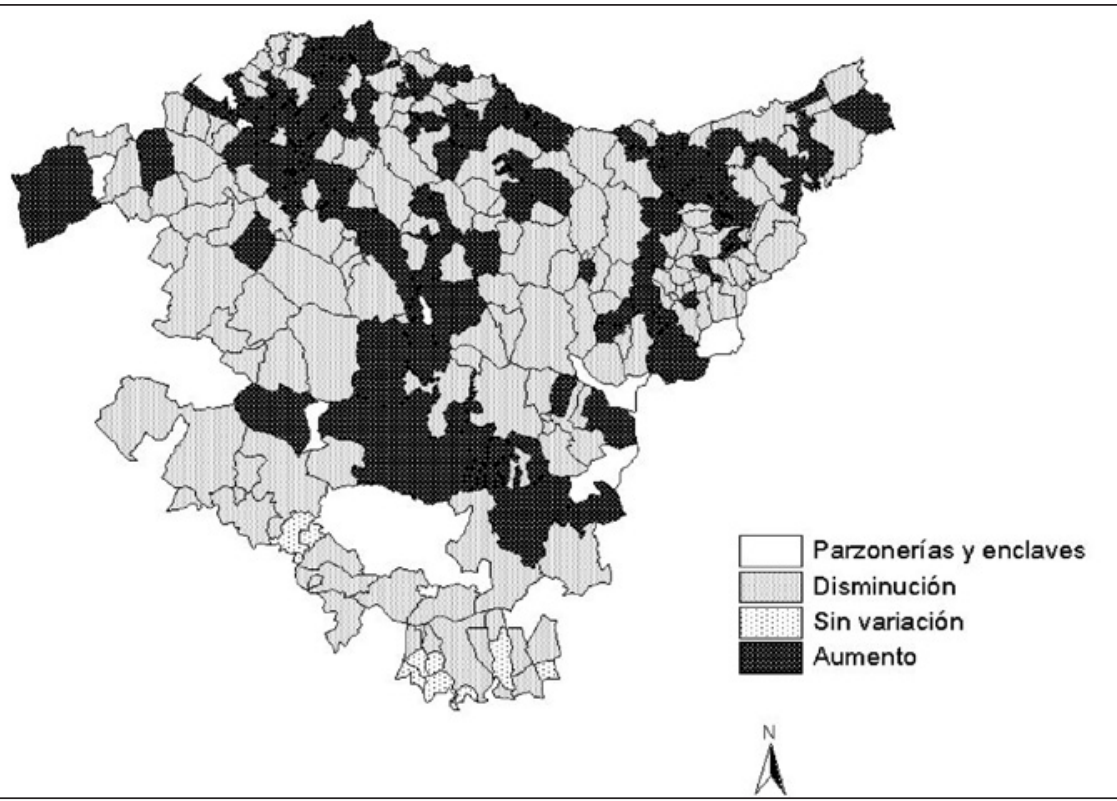

Fuente: INE, Censo Agrarios. 
Su reparto geográfico resulta muy aleatorio, ya que se entremezclan los términos con ganancias con los que decrecen, y no resulta factible establecer alguna pauta espacial que pueda ser explicada. La única consecuencia práctica es que las consecuencias del abandono de la cabaña ovina, aun no siendo muy acusado hasta el momento, afectan a la mayor parte del territorio vasco, independientemente de su entorno geoecológico.

\section{VALORACIONES FINALES}

Se ha constatado un descenso del número de explotaciones ganaderas, así como una disminución del número de cabezas. Las principales pérdidas en uno y otro caso corresponden en general a las explotaciones de menor tamaño de SAU, mientras se confirma un proceso de concentración de los ganados en las explotaciones de mayor base territorial. Esta intensa reestructuración del sector ganadero debería tener un impacto en los usos del suelo y en el paisaje. Veamos qué ha sucedido en estos diez años con las superficies dedicadas a la alimentación del ganado.

Tabla 7

EVOLUCIÓN DE LA SUPERFICIE DE CULTIVOS FORRAJEROS Y DE PASTOS PERMANENTES (HA) EN EXPLOTACIONES CON MÁS DE 1 HECTÁREA EN EL PAÍS VASCO.

\begin{tabular}{|l|c|c|}
\hline & Cultivos forrajeros & Pastos permanentes \\
\hline 1999 & 2.467 & 170.634 \\
\hline 2009 & 5.941 & 111.196 \\
\hline
\end{tabular}

Fuente: INE, Censos Agrarios.

A pesar del aumento de los cultivos forrajeros, que parece indicar una tendencia reciente a una intensificación en la alimentación del ganado, el balance total de las tierras dedicadas a ese fin ha registrado, según los datos censales, un retroceso muy considerable. No obstante estos datos hay que tomarlos con cautela. El considerable descenso de la superficie de pastos permanentes no tiene por qué ser interpretado necesariamente como un cambio de ocupación del suelo, sino que en gran parte al menos se debería a una pérdida de función ganadera de esos espacios, que sin embargo conservan por ahora su vegetación herbácea, al haber disminuido el montante de los ganados. Si se recurre a otras fuentes estadísticas como el Anuario de Estadística los valores son bien distintos.

Tabla 8

EVOLUCIÓN DE LA SUPERFICIE DE PRADOS Y PASTIZALES (HA) EN EL PAÍS VASCO, 1999 Y 2009

\begin{tabular}{|l|c|c|}
\hline & 1999 & 2009 \\
\hline Prados y pastizales & 150.630 & 151.729 \\
\hline
\end{tabular}

Fuente: Ministerio de Agricultura, Alimentación y Medio Ambiente, Anuarios de Estadística.

Según esta fuente la superficie de herbazales permanece inalterada a lo largo de esos diez años. No habría habido cambios en el uso y daría fuerza a la interpretación funcional 
que se ha realizado de los resultados censales. En un trabajo anterior Porcal, Galdos y Ruiz (2009) realizan un estudio sobre los cambios de ocupación del suelo en las áreas de montaña del País Vasco en base a la información suministrada por el Inventario Forestal del año 2005 y las imágenes del proyecto CORINE de los años 1987 y 2000. La dinámica de cambio en estas montañas, sobre todo en las de carácter atlántico, es mucho más viva de la que podría suponerse. Se calcula que en los 13 años que median entre 1987 y 2000 el 18,5\% de la superficie de la montaña atlántica ha modificado su cobertura. En su mayoría obedece a transformaciones en la cubierta forestal arbolada y sobre todo en relación con los cultivos forestales de rápido crecimiento. Pero también se registran modificaciones en ámbitos ganaderos. Se comprueba un retroceso superficial de prados y praderas, no de gran envergadura, a favor de la expansión de la forestación, del matorral boscoso de transición, y de la urbanización, sobre todo de baja densidad, e infraestructuras de transporte (Alberdi, 2001a). Por el contrario también se registra un ligero aumento del espacio ocupado por los pastizales. Los cambios más frecuentes en la vertiente atlántica respecto de la mediterránea no se achacan sólo a factores de carácter físico, sino que adquieren protagonismo otros factores más ligados a las estructuras agrarias, como el predominio de la propiedad privada, con un uso más intensivo del suelo que busca obtener rendimientos económicos a corto plazo, a la elevada densidad de población y la compacta red urbana.

Con todo parece claro que el declive de la ganadería no ha llegado a transformar por el momento de una forma radical, aunque sí comprobable, el paisaje de la campiña vascoatlántica. Si esto es así, cabe hacerse la siguiente pregunta ¿es posible un paisaje ganadero sin ganaderos? En primer lugar hay que remarcar que muchos de los ganaderos que se mantienen hacen un uso más extensivo del territorio, aún a costa de que una parte de los espacios dedicados a la alimentación del ganado permanezcan infrautilizados. La extensificación se relaciona con la mayor resistencia del ganado lanar a su desaparición y a la conversión de una parte notable de la producción láctea en cárnica. Aunque como ya se ha expuesto con anterioridad este concepto de extensificación oculta en muchos casos un semiabandono o una reducida utilización de los recursos (Murua et al., 2012).

Como un acicate para avivar el interés de los ganaderos para su continuidad hay que mencionar el papel de las políticas llevadas a cabo en Europa y en el País Vasco para fomentar las producciones de calidad. Por lo que se refiere a productos ganaderos existen varios distintivos de calidad: Denominación de Origen del queso Idiazabal, los lábeles vascos de carne de vacuno Euskal Okela y de cordero lechal Euskal Esne Bildotsa. A finales de 2011 estaban registrados 1.190 productores de carne de vacuno, 256 de cordero lechal y 95 pequeños elaboradores de queso Idiazabal. Ahora bien, según la evolución reciente de los registros, acogerse a un distintivo de calidad agroalimentaria tampoco garantiza la permanencia de las explotaciones ganaderas. De hecho cada vez son menos los productores que se integran dentro de los distintivos certificados por el Gobierno Vasco.

Por otro lado la propia dimensión territorial reducida del caserío vasco no permite grandes variaciones en su organización interna, tal como ha venido sucediendo en otras fases históricas anteriores. Pero aquí entran en juego otros factores no relacionados directamente con la estructura agraria, ni con la actividad agraria como tal actividad económica. Se ha comprobado cómo muchas explotaciones ganaderas siguen subsistiendo a pesar de su marginalidad económica, dentro de lo que se ha catalogado como agricultura de ocio. 
En efecto numerosas pequeñas explotaciones, cuya renta básica procede de otros sectores ajenos a lo agrario, tienen diseñada una estrategia de gestión de su espacio vinculada al paso de la SAU (Superficie Agrícola Utilizada) al SAU (Superficie Apta para Urbanizar), como gráficamente ha descrito Ainz (1999). Esto significa que a muchas parcelas particulares sus propietarios les conceden un valor económico potencial ligado a su posible urbanización, incluso aquellas que se encuentran más alejadas de los actuales entornos urbanos o en zonas declaradas no urbanizables, circunstancia que puede ser modificada en el futuro. Para que las parcelas de praderío conserven su valor potencial es aconsejable que se mantengan limpias y cuidadas, por lo que el propietario cría unas pocas cabezas dirigidas esencialmente a ese fin. Su forestación supondría una hipoteca a medio o largo plazo que tampoco compensa en ciclos de baja cotización de la madera de coníferas de rápido crecimiento. En este comportamiento también influye el prurito profesional de estos ganaderos marginales que siguen conservando una vocación agraria y un orgullo por el buen cuidado de las tierras (Ainz, 2001a). Esta misma estrategia también perdura en antiguas explotaciones que han abandonado la actividad y en las que el caserío ha dejado de ejercer la función agraria para convertirse en vivienda privilegiada en un entorno rural. Estos caseríos, muchas veces totalmente remozados o incluso de nueva planta, aparecen rodeados por las antiguas tierras de labranza, hace ya tiempo convertidas en praderas. El interés de sus propietarios es que este entorno se mantenga, lo que no sucedería si se acometiese una repoblación que acabase «comiéndose» a la vivienda. Para ello se recurre a otras tácticas como la cesión oral y sin contrato del aprovechamiento de las praderas a otros ganaderos que permanecen y necesitan más espacio, aunque sea de esta forma precaria y nunca definitiva. Lo que resulta más dudoso es que esta situación de equilibrio de usos se mantenga a medio o largo plazo, conforme se vayan consumando los relevos generacionales (Alberdi, 2001). Lo que sí parece claro, tal como ha venido sucediendo hasta ahora, es que una parte esencial de los factores que guíen la evolución de las explotaciones agrarias y las estrategias de sus gestores va a provenir del exterior del propio sector, máxime en unas explotaciones que generan tan poco trabajo en su estado actual: las explotaciones ganaderas justo reclaman de media 1 UTA (Unidad de Trabajo Año), mientras que las mixtas ganaderoagrícolas ni siquiera llegan a esa magnitud.

\section{BIBLIOGRAFÍA}

AINZ IBARRONDO, M. J. (1999) «La proyección espacial del caserío vasco: desde su antigua función de unidad agraria plurifuncional a la más nueva de suelo apto para urbanizar». Boletín de la Asociación de Geógrafos Españoles, n 27, pp. 47-66.

AINZ IBARRONDO, M. J. (2001) El caserío vasco en el país de las industrias. Ministerio de Agricultura, Pesca y Alimentación, Madrid.

AINZ IBARRONDO, M. J. (2001a) «El sentido de las praderas vasco-atlánticas», en Manero, F. (coord.) Espacio natural y dinámicas territoriales: homenaje al Dr. Jesús García Fernández, Universidad de Valladolid, Valladolid, pp. 397-408.

ALBERDI COLLANTES, J. C. (2001) De caserío agrícola a vivienda rural: evolución de la función agraria en la comarca de Donostia-San Sebastián. Gobierno Vasco, Departamento de Agricultura y Pesca, Vitoria-Gasteiz. 
ALBERDI COLLANTES, J. C. (2001a) «Reestructuración agraria y abandono de usos: el caserío vasco». Investigaciones Geográficas, n² 26, pp. 135-150.

ALBERDI COLLANTES, J. C. (2002) «Desagrarización del caserío y abandono forestal». Sancho el Sabio, no 16 , pp. 11-30.

ARNALTE, E., ORTIZ, D. y MORENO O. M. (2012) Lecciones de política rural: la política rural de la Unión Europea y su aplicación en España. Universitat Politècnica de Valencia, Valencia.

BASERRI BIZIA (2011) «Son necesarias medidas estructurales de apoyo al sector lácteo en Euskal Herria», Revista del Sindicato Agrario EHNE-Bizkaia, , n ${ }^{\circ}$, pp. 14-15.

BASERRI BIZIA (2012) «El precio de las materias primas, un escándalo», Revista del Sindicato Agrario EHNE-Bizkaia, no 32, pp. 12-13.

BERGA, A. M., ALÉ, R. y SÁEZ, E. (2008) «La nueva Política Agraria Común. La nueva situación de las producciones ganaderas», en Sáez, E. (coord.) Desarrollo sostenible, Diputación Provincial de Huesca, pp. 41-54.

BOURNARIS, Th. y MANOS, B. (2012) «European Union agricultural scenarios' impacts on social sustainability of agricultural holdings». International Journal of Sustainable Development and World Ecology, vol. 19-5, pp. 426-432.

BRADY, M., SAHRBACHER, C., KELLERMANN, K. et al. (2012) «An agent-based approach to modeling impacts of agricultural policy on land use, biodiversity and ecosystem services». Landscape Ecology, vol. 27-9, pp. 1363-1381.

CAMÓS, M. (2006) «La política de desarrollo rural en el marco de la política agraria común». Cuadernos de Derecho Local, nº 10, pp. 22-34.

CARO BAROJA, J. (1971, $3^{\mathrm{a}}$ ed.) Los vascos. Istmo, Madrid.

CASCOS MARAÑA, C. (2011) «La simplificación y la uniformidad crecientes en los paisajes ganaderos de la montaña húmeda de Castilla y León. El ejemplo de Babia y Luna». Polígonos, $\mathrm{n}^{\circ}$ 21, pp. 149-178.

CEJUDO, E., y MAROTO, J. C. (2010) «La reforma de la PAC 2003: desacoplamiento, condicionalidad, modulación, desarrollo rural». Scripta Nova, vol. XIV, nº 318.

CORBERA MILLÁN, M. (2006) «La resistencia de un sistema milenario de trashumancia ganadera de corto recorrido en el valle de Cabuérniga». Scripta Nova, vol. 10, no 218.

CORBERA MILLÁN, M. (2008) «El proceso de colonización y la construcción del paisaje en los Montes de Pas». Eria, nº 77, pp. 293-314.

DOMÍNGUEZ MARTÍN, R. (ed.) (1996) La vocación ganadera del norte de España. Del modelo tradicional a los desafíos del mercado mundial. Ministerio de Agricultura, Pesca y Alimentación. Serie Estudios, Madrid.

ELÍAS PASTOR, J.M.; FUENTES OCHOA, C. (2001) «Efectos de la ganadería en el paisaje de 1s Sierra riojana». Zubía, no 13, pp. 139-156.

ETXEZARRETA, M. (1977) El caserío vasco. Fundación C. de Iturriaga y M. de Dañobeitia, Bilbao.

GARCÍA FERNÁNDEZ, J. (1975) Organización del espacio y economía rural en la España Atlántica. Ed, Siglo XXI, Madrid.

GASCÓ, J. M., NAREDO, J. M. y PARRA, F. (2002) «Sistema rural. La modernización de 1 agricultura española y sus repercusiones ecológicas», en Naredo J. M. y Parra, F. (coords.) Situación diferencial de los recursos españoles, Fundación César Manrique, Teguise, Lanzarote, pp. 229-252. 
HATNA, E. y BAKKER, M. M. (2011) «Abandonment and Expansion of Arable Land in Europe». Ecosystems, n 14 , pp. 720-731.

KEENLEYSIDE, C., TUCKER, G. (2010) Farmland Abandonment in the EU: an Assessment of Trends and Prospects. Institute for European Environmental Policy, http://www. ieep.eu/assets/733/Farmland_abandonment_in_the_EU_-_assessment_of_trends_and_ prospects___FINAL_15-11-2010_.pdf

LLORENTE PINTO, J.M. (2011) «Dehesas y paisajes adehesados en Castilla y León». Polígonos, $\mathrm{n}^{\circ} 21,179-203$.

MALAGÓN, E. (2012) «La Política Agraria Común y las zonas de montaña». Ingurugiroa eta zuzenbidea-Ambiente y derecho, $\mathrm{n}^{\mathrm{o}}$ 10, pp. 13-26.

MICHEL, M. (2004) El pino radiata («Pinus radiata»D. Don) en la historia forestal de la Comunidad Autónoma de Euskadi. Servicio Central de Publicaciones del Gobierno Vasco, Vitoria-Gasteiz.

MURUA, J. R., EGUIA, B., MALAGÓN, E., y AlBIAC, J. (2006) Coste de la no agricultura en el País Vasco. Departamento de Agricultura, Pesca y Alimentación, Servicio Central de Publicaciones del Gobierno Vasco, Vitoria-Gasteiz.

MURUA, J.R.; ASTORKIZA, I.; EGUIA, B. (2012) «Abandono e infrautilización del suelo agrario en la Cornisa Cantábrica. El caso del País Vasco». Revista Española de Estudios Agrosociales, $\mathrm{n}^{\circ}$ 231, pp. 63-91.

NAREDO, J. M. (1996) La evolución de la agricultura en España (1940-1990). Universidad de Granada, Granada.

PARRA, F. (2002) «Sistema rural. La modernización de la agricultura española y sus repercusiones ecológicas». En Naredo, J. M. y Parra F. (eds.) Situación diferencial de los recursos naturales españoles, Fundación César Manrique, Madrid, pp. 229-252.

PIORR, A., UNGARO, F., CIANCAGLINI, A, HAPPE, K., SAHRBACHER, A., SATTLER, C., UTHES, S., y ZANDER. P. (2009) «Integrated assessment of future CAP policies: land uses changes, spatial patterns and targeting». Environmental Science \& Policy, $\mathrm{n}^{\circ} 12$ (8), pp. 1122-1136.

PORCAL GONZALO, M. C., GALDOS URRUTIA, R., RUIZ URRESTARAZU (2009) «Dinámica paisajística de las áreas de montaña del País vasco a través de los cambios en la ocupación del suelo». XXI Congreso de Geógrafos Españoles. Geografía, Territorio y Paisaje: el estado de la cuestión, Universidad de Castilla- La Mancha, pp. 1307-1322..

RUIZ URRESTARAZU, E., GALDOS URRUTIA, R. (2005) «Reflexiones sobre la desestructuración del caserío vasco». Investigaciones Geográficas, n³ 38, pp. 79-91.

RUIZ URRESTARAZU, E., GALDOS URRUTIA, R. (2011) «La crisis que no cesa. Perspectiva territorial del abandono de explotaciones agrarias en el País Vasco (1999-2009)». Lurralde: investigación y espacio, $\mathrm{n}^{\mathrm{o}} 34$, pp. 301-313.

SILVA PÉREZ, R. (2010) «La dehesa vista como paisaje cultural. Fisonomías, funcionalidades y dinámicas históricas». Eria, nº 82, pp. 143-157.

SINEIRO, F., LÓPEZ, E., RIBAS A. y LORENZANA R. (2006) «Los factores explicativos del ajuste estructural reciente en la ganadería bovina de la cornisa cantábrica», en Arnalte, E. (coord.) Políticas agrarias y ajuste estructural en la agricultura española, Ministerio de Agricultura, Pesca y Alimentación, Madrid, pp. 291-326. 
TZANOPOULOS, J., JONES. P. J., MORTIMER, S. R. (2012) «The implications of the 2003 Common Agricultural Policy reforms for land-use and landscape quality in England». Landscape and Urban Planning, vol. 108-1, pp. 39-48.

VALLE BUENESTADO, B. (1995) «Los paisajes agrarios del norte de Córdoba», en Propiedad, actividad agraria y medio ambiente en España y América Latina. Actas del VII Coloquio de Geografía Rural (ponencias y excursiones), pp. 235-260.

VILA i SUBIRÓS, J.; RIBAS i PALOMA, A.; VARGA i LINDE, D.; LLAUSÀS PASCUAL, A. (2009) «Medio siglo de cambios paisajísticos en la montaña mediterránea: percepción y valoración social del paisaje en la alta Garrotxa (Girona)». Pirineos, n 164, pp. 69-92. 